\title{
The Smallest Enclosing Ball of Balls: Combinatorial Structure and Algorithms ${ }^{* \dagger}$
}

\author{
Kaspar Fischer \\ Institut für Theoretische Informatik, ETH Zürich \\ ETH Zentrum, CH-8092 Zürich, Switzerland \\ fischerk@inf.ethz.ch
}

\author{
Bernd Gärtner \\ Institut für Theoretische Informatik, ETH Zürich \\ ETH Zentrum, CH-8092 Zürich, Switzerland \\ gaertner@inf.ethz.ch
}

\begin{abstract}
We develop algorithms for computing the smallest enclosing ball of a set of $n$ balls in $d$-dimensional space. Unlike previous methods, we explicitly address small cases $(n \leq d+1)$, derive the necessary primitive operations and show that they can efficiently be realized with rational arithmetic. An exact implementation (along with a fast ${ }^{1}$ and robust floating-point version) is available as part of the CGAL library. ${ }^{2}$

Our algorithms are based on novel insights into the combinatorial structure of the problem. As it turns out, results for smallest enclosing balls of points do not extend as one might expect. For example, we show that Welzl's randomized linear-time algorithm for computing the ball spanned by a set of points fails to work for balls. Consequently, David White's adaptation of the method to the ball case - as the only available implementation so far it is mentioned in many link collections - is incorrect and may crash or, in the better case, produce wrong balls.

In solving the small cases we may assume that the ball centers are affinely independent; in this case, the problem is surprisingly well-behaved: via a geometric transformation and suitable generalization, it fits into the combinatorial model of unique sink orientations whose rich structure has recently received considerable attention. One consequence is that Welzl's algorithm does work for small instances; moreover, there is a wide variety of pivoting methods for unique sink orientations which have the potential of being fast in practice even for high dimensions.

\footnotetext{
*Partly supported by the IST Programme of the EU and the Swiss Federal Office for Education and Science as a Shared-cost RTD (FET Open) Project under Contract No IST-2000-26473 (ECG - Effective Computational Geometry for Curves and Surfaces).

${ }^{\dagger}$ Supported by the Berlin/Zürich Joint Graduate Program "Combinatorics, Geometry, and Computation" (CGC).

${ }^{1}$ For $d=3$, a set of $1,000,000$ balls is processed in less than two seconds on a modern PC.

${ }^{2}$ See http://www.cgal.org.
}

Permission to make digital or hard copies of all or part of this work for personal or classroom use is granted without fee provided that copies are not made or distributed for profit or commercial advantage and that copies bear this notice and the full citation on the first page. To copy otherwise, to republish, to post on servers or to redistribute to lists, requires prior specific permission and/or a fee.

SoCG'03, June 8-10, 2003, San Diego, California, USA.

Copyright 2003 ACM 1-58113-663-3/03/0006 ...\$5.00.
As a by-product, we show that the problem of finding the smallest enclosing ball of balls is computationally equivalent to the problem of finding the minimum-norm point in the convex hull of a set of balls.

\section{Categories and Subject Descriptors}

F.2.1 [Analysis of Algorithms and Problem Complexity]: Nonnumerical Algorithms and Problems-Geometrical problems and computations; I.3.5 [Computer Graphics]: Computational Geometry and Object Modeling; G.2.1 [Discrete Mathemetics]: Combinatorics-Combinatorial algorithms

\section{General Terms}

Algorithms

\section{Keywords}

minimum volume sphere, minimal covering ball, distance to convex hull, unique sink orientation

\section{INTRODUCTION}

In this paper we study the problem of finding the closed ball of smallest radius (equivalently, smallest volume) which contains a given set of $n$ closed balls in $d$-dimensional Euclidean space. This problem - which we denote by SEBBgeneralizes the well-understood problem SEBP of finding the smallest enclosing ball of $n$ points.

Applications include collision detection, the computation of bounding sphere hierarchies for clustering or efficient rendering of complex scenes, culling (e.g. for visualization of molecular models [21]), automated manufacturing [10] and similarity search in feature spaces [13].

The SEBB problem can be solved in time $O(n)$ for fixed dimension $d$, where all known bounds are exponential in $d$. For SEBP, the first (deterministic) linear-time algorithmbased on the prune-and-search paradigm - is due to Megiddo $[15,16]$, with subsequent improvements by Dyer [5]; Welzl's randomized algorithm [20] is the first 'practical' algorithm to achieve this bound. Extending the applicability of pruneand-search, Megiddo [17] and Dyer [6] later showed that the $O(n)$ bound also applies to SEBB. Linear-time algorithms for SEBB also result from the observation that the problem is LP-type [14], in which case generic $O(n)$-methods for this problem class can be used $[14,3]$.

When it comes to actual implementations, none of the above methods work out of the box; the prune-and-search 
approaches require routines for solving systems of constantdegree algebraic equations, while the LP-type approach asks us to provide a method of computing SEBB for small instances $(n \leq d+2)$. To the best of our knowledge, there is no published code that addresses these primitive operations. Restricting attention to the SEBP problem, we also have Welzl's algorithm at our disposal which has the attractive feature that the primitive operations are very easy to implement (compute the unique circumsphere of an affinely independent set of points). It is therefore tempting to generalize Welzl's algorithm to the case of balls and hope for a similarly simple primitive. This is exactly the approach of David White's algorithm, so far the only code we know of to solve the SEBB problem. Links to White's code appear in many web directories of computational geometry software.

Unfortunately, this extension of Welzl's algorithm does not work, as we show in this paper. The reason is that the correctness proof is based on a lemma [20, Lemma 1] which does not extend to the balls case. We exhibit concrete, nondegenerate examples of input balls for which Welzl's algorithm (and in particular, White's code) computes balls which are too large, or even reaches an undefined state.

Having said this, it might come as a surprise that Welzl's method does extend to the case where the ball centers are affinely independent, and this becomes an important case, if we want to solve the small instances within the LP-type algorithm mentioned above. Unfortunately, already in the case of points there are inputs (e.g. the vertices of a regular simplex) for which Welzl's algorithm has complexity $\Omega\left(2^{d}\right)$, with the effect that the small cases become the limiting factor already for moderately high dimensions. Given the additional fact that the small instances in the LP-type algorithm have size up to $d+2$ and are in general not affinely independent, our implementation does not use Welzl's method but solves the small cases by an exhaustive search. In this approach, the primitive operations require some care but are otherwise as easy as in Welzl's method; the resulting code is very fast for dimensions up to 10 .

For dimensions beyond that, it is known that the performance can be improved for the case of points: in theory, there are subexponential methods [7]; there is also a very practical approach that reduces the complexity to $O\left((3 / 2)^{d}\right)$ [9], and using the recent concept of unique sink orientations, this can be improved even further [19]. On the practical side, the unique sink approach allows for pivoting methods for which we may not be able to give performance guarantees, but which have the potential of being fast for almost any input. Even though these improved techniques require affine independence, the gain in runtime would justify an embedding of our (at most $(d+2)$-element) pointset in dimension $d+1$, followed by a symbolic perturbation to attain affine independence.

However, for all these techniques to extend to the case of balls, a generalization of the SEBB problem is necessary. To explain the issue, let us look at the basic objects handled by the extension of Welzl's method to the balls case: the generic recursive call of the algorithm computes $\operatorname{MB}(U, V)$, the smallest ball containing some set of balls $U$, under the additional restriction that this ball has to be tangent to all balls in $V \subseteq U$. The primitive operation is the computation of $\operatorname{mB}(V, V)$. While this ball exists and is unique if $V$ is a set of points (recall that we assume affine indepedence), the general case is less well-behaved. There might be no enclosing ball tangent to all balls in $V$; it can even happen that $V$ does not allow a tangent ball at all (Figure 1).

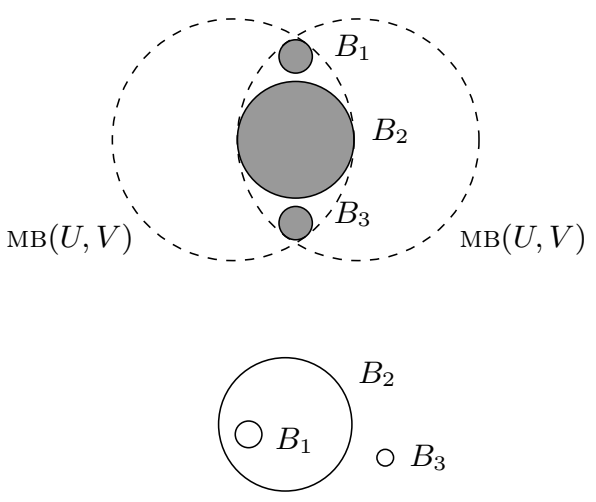

Figure 1: $\operatorname{\mathrm {B}}(U, V)$ need neither be unique (top) nor exist (bottom): set $U=V=\left\{B_{1}, B_{2}, B_{3}\right\}$.

While Welzl's algorithm has the nice self-stabilizing property (only in the balls case, this requires affine independence) of considering only pairs $(U, V)$ for which $\operatorname{MB}(U, V)$ exists and is unique, the other approaches mentioned above potentially consider arbitrary pairs $(U, V)$. In section 4 , we reduce SEBB to finding $\operatorname{MB}_{p}(U, V)=\operatorname{MB}(U \cup\{p\}, V \cup\{p\})$, i.e. the smallest ball containing the balls $U$ while being tangent to the balls $V$ and some point $p$, and introduce a 'generalized' ball $\operatorname{GMB}_{p}(U, V)$ whose existence and uniqueness holds for all parameters $(p, U, V)$. Moreover, if $\operatorname{MB}_{p}(U, V)$ exists then it coincides with $\operatorname{GMB}_{p}(U, V)$.

In order to obtain $\operatorname{GMB}_{p}(U, V)$, we first use inversion, a geometric transformation which in some sense allows us to 'linearize' the problem; in this dual formulation, we are faced with the problem of finding the lowest point in a cyclinder, subject to linear (in)equality constraints. Adding another dimension makes sure that this point always exists and is unique. Moreover, the problem of finding it can be written as a convex program to which we subsequently apply the Karush-Kuhn-Tucker optimality conditions. From these, our structural results are easily derived.

\section{BASICS}

A $d$-dimensional ball with center $c \in \mathbb{R}^{d}$ and radius $\rho \in \mathbb{R}$ is the set $B(c, \rho)=\left\{x \in \mathbb{R}^{d} \mid\|x-c\|^{2} \leq \rho^{2}\right\}$. We denote the center and radius of a ball $B$ by $c_{B}$ and $\rho_{B}$, respectively. If $\rho_{B} \geq 0$, we call the ball positive, if $\rho_{B} \leq 0$, we say it is negative. Where not explicitly stated otherwise, our balls under consideration are assumed to be positive. We take for granted the fact that two balls $B(c, \rho)$ and $B\left(c^{\prime}, \rho^{\prime}\right)$ (where we assume $|\rho| \geq\left|\rho^{\prime}\right|$ w.l.o.g.) are tangent if and only if $\left\|c-c^{\prime}\right\|=|\rho|+\left|\rho^{\prime}\right|$, in which case we speak of external tangency, or $\left\|c-c^{\prime}\right\|=|\rho|-\left|\rho^{\prime}\right|$, in which case $B\left(c^{\prime}, \rho^{\prime}\right) \subseteq B(c, \rho)$ is said to be internally tangent to $B(c, \rho)$. For a set $U$ of $n$ balls in $\mathbb{R}^{d}$, we define the miniball $\operatorname{MB}(U)$ to be the ball of minimal radius which encloses all balls in $U$. A standard compactness argument, together with some properties of convex combinations of balls [20], gives:

Lemma 2.1. $\operatorname{mB}(U)$ exists and is unique. 
The following lemma is based on a similar statement for points due to Seidel [18]. Recall that a point $q \in \mathbb{R}^{d}$ lies in the convex hull conv $(P)$ of a point set $P \subseteq \mathbb{R}^{d}$ if and only if $\min _{p \in P}(p-q)^{T} u \leq 0$ for all unit vectors $u$.

Lemma 2.2. Let $B$ be internally tangent to the balls in $V$. Then $B=\operatorname{MB}(V)$ if and only if $c_{B} \in \operatorname{conv}\left(\left\{c_{D} \mid D \in V\right\}\right)$.

Proof. $(\Leftarrow)$ Assume $B \neq \mathrm{MB}(V)$, i.e. there exists an enclosing ball $B^{\prime}$ with radius $\rho_{B}^{\prime}<\rho_{B}$. Write its center as $c_{B}^{\prime}=c_{B}+\lambda u$ for some unit vector $u$ and $\lambda>0$. Then the distance from $c_{B}^{\prime}$ to the farthest point in the ball $D$ is

$$
\begin{aligned}
\delta_{D} & =\left\|c_{B}^{\prime}-c_{D}\right\|+\rho_{D} \\
& =\sqrt{\left(c_{B}+\lambda u-c_{D}\right)^{T}\left(c_{B}+\lambda u-c_{D}\right)}+\rho_{D} \\
& =\sqrt{\left\|c_{B}-c_{D}\right\|^{2}+\lambda^{2} u^{T} u-2 \lambda\left(c_{D}-c_{B}\right)^{T} u}+\rho_{D} \\
& =\sqrt{\left(\rho_{B}-\rho_{D}\right)^{2}+\lambda^{2}-2 \lambda\left(c_{D}-c_{B}\right)^{T} u}+\rho_{D} .
\end{aligned}
$$

Since $B^{\prime}$ is enclosing, we have $\rho_{B}^{\prime} \geq \max _{D \in V} \delta_{D}$. Furthermore, the observation preceding the lemma yields $D \in V$ such that $\left(c_{D}-c_{B}\right)^{T} u \leq 0$, for $c_{B}$ lies in the convex hull of the centers of $V$. Consequently,

$$
\delta_{D}>\sqrt{\left(\rho_{B}-\rho_{D}\right)^{2}}+\rho_{D}=\rho_{B}>\rho_{B}^{\prime}
$$

by equation (1), a contradiction. Conversely, suppose that $c_{B}$ does not lie in the convex hull of the centers of $V$. By the above observation there exists a vector $u$ of unit length with $\left(c_{D}-c_{B}\right)^{T} u>0$ for all $D \in V$. So consider the point $c_{B}^{\prime}=c_{B}+\lambda u$, for some very small $\lambda>0$. According to (1), $\delta_{D}<\left(\rho_{B}-\rho_{D}\right)+\rho_{D}=\rho_{B}$ for all $D$, and consequently, the ball $B^{\prime}$ with center $c_{B}^{\prime}$ and radius $\max _{D} \delta_{D}<\rho_{B}$ is enclosing, contradiction.

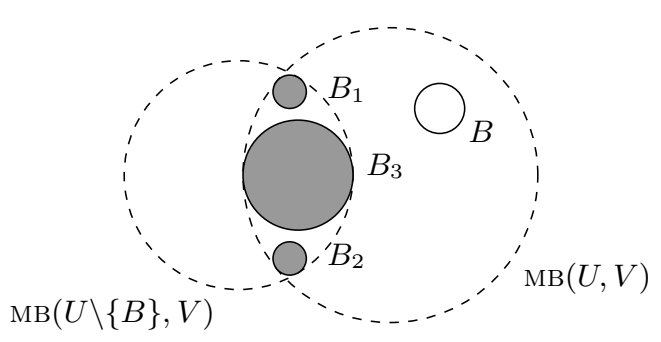

Figure 2: Ball $B$ cannot be dropped although it is properly contained in $\mathrm{MB}(U, V)$.

Another property our algorithm from section 3 makes use of is the following (proof omitted), which does not generalize to $\operatorname{mB}(U, V)$ (refer for instance to Figure 2, where $\operatorname{mB}(U, V) \neq$ $\operatorname{MB}(U \backslash\{B\}, V)$, for $V=\left\{B_{1}, B_{2}, B_{3}\right\}$ and $\left.U=V \cup\{B\}\right)$.

LEMmA 2.3. If ball $B \in U$ is properly contained in $\mathrm{MB}(U)$, then $\operatorname{MB}(U)=\operatorname{MB}(U-\{B\})$.

Motivited by this statement, we call a set $U^{\prime} \subseteq U$ a support set of $U$ if all balls in $U^{\prime}$ touch the boundary of $\mathrm{MB}(U)$ (that is, if they are internally tangent to $\operatorname{MB}(U))$ and $\operatorname{MB}(U)=$ $\operatorname{MB}\left(U^{\prime}\right)$. An inclusion-minimal support set of $U$ is called $b a$ sis of $U$. The following lemma, together with the preceding one, shows that the miniball is determined by a support set of size at most $d+1$.
Lemma 2.4. Let $U$ be a set of at least $d+1$ balls in $\mathbb{R}^{d}$. Then there exists a subset $U^{\prime} \subseteq U$ of $d+1$ elements such that $\operatorname{MB}(U)=\operatorname{MB}\left(U^{\prime}\right)$.

Proof. Let $D=\operatorname{MB}(U)$ be the smallest enclosing ball of $U$ and consider $I=\bigcap_{B \in U} B\left(c_{B}, \rho_{D}-\rho_{B}\right)$. Observe that $B\left(c_{B}, \rho_{D}-\rho_{B}\right)$ is the set of all centers which admit a ball of radius $\rho_{D}$ that encloses $B$. By the existence and uniqueness of $\operatorname{MB}(U), I$ thus contains exactly one point, namely $c_{D}$. It follows that $\bigcap_{B \in U}$ ri $B\left(c_{B}, \rho_{D}-\rho_{B}\right)=\emptyset$, where ri $B$ denotes the interior of a ball $B$. Helly's Theorem [4] yields a set $U^{\prime} \subseteq U$ of $d+1$ elements such that $\bigcap_{B \in U^{\prime}}$ ri $B\left(c_{B}, \rho_{D}-\right.$ $\left.\rho_{B}\right)=\emptyset$. Consequently, no ball of radius $<\rho_{D}$ encloses the balls $U^{\prime}$, and thus $\operatorname{\mathrm {mB}}(U)$ and $\mathrm{mB}\left(U^{\prime}\right)$ have the same radius. This however implies $\operatorname{MB}(U)=\operatorname{MB}\left(U^{\prime}\right)$, since we would have found two different miniballs of $U^{\prime}$ otherwise.

Lemma 2.5. The centers of a basis $V$ of $U$ are affinely independent.

Proof. By Lemma 2.2, the center $c_{D}$ of $D=\operatorname{MB}(V)$ can be written as $c_{D}=\sum_{B \in V} \lambda_{B} c_{B}$ for coefficients $\lambda_{B} \geq 0$ summing up to 1 . Observe that $\lambda_{B}>0$ in this summation, by minimality of $V$ and Lemma 2.2. So suppose that the centers $\left\{c_{B} \mid B \in V\right\}$ are affinely dependent. Then there exist coefficients $\mu_{B}$, not all zero, such that $\sum_{B \in V} \mu_{B} c_{B}=\mathbf{0}$ and $\sum \mu_{B}=0$. Consequently,

$$
c_{D}=\sum_{B \in V} c_{B}\left(\lambda_{B}+\alpha \mu_{B}\right) \quad \text { for any } \alpha \in \mathbb{R} .
$$

Change $\alpha$ continuously from 0 on and stop as soon as $\lambda_{B}+$ $\alpha \mu_{B}=0$ for some $B$. At this moment all coefficients $\lambda_{B}^{\prime}=$ $\lambda_{B}+\alpha \mu_{B}$ of the combination (2) are strictly positive with the exception of $\lambda_{B}^{\prime}$, a contradiction to the minimality of $V$.

\section{ALGORITHMS}

Our algorithms are combinatorial in nature and are based on the following generalization of $\operatorname{mB}(U)$. For sets $U \supseteq V$ of balls, denote by в $(U, V)$ the set of balls $B$ which contain the balls $U$ and are internally tangent to (at least) the balls in $V$. Moreover, define $\operatorname{MB}(U, V)$ to be the (set of) smallest ball(s) in $\mathrm{B}(U, V)$. Notice that $\operatorname{MB}(U, V)$ need neither exist nor be unique (Figure 1), and that $\operatorname{MB}(U)=\operatorname{MB}(U, \emptyset)$. Some properties of $\operatorname{MB}(U, V)$ are developed in Sections 4 and 5 ; for the moment, we content ourselves with the following.

Lemma 3.1. Let $U^{\prime}$ be a basis of $U$. Then $\operatorname{MB}\left(U^{\prime}, U^{\prime}\right)$ is unique, coincides with $\mathrm{MB}(U)$ and can be computed in $O\left(d^{3}\right)$.

Proof. As a basis, $U^{\prime}$ satisfies $\operatorname{MB}\left(U^{\prime}\right)=\operatorname{MB}(U)$. Futhermore, the balls $U^{\prime}$ must be tangent to $\mathrm{MB}(U)$ by Lemma 2.3. Consequently, $\operatorname{MB}\left(U^{\prime}\right)=\operatorname{MB}\left(U^{\prime}, U^{\prime}\right)$, with the uniqueness following from Lemma 2.1. Let $U^{\prime}=\left\{B_{1}, \ldots, B_{m}\right\}$ and observe that $B(c, \rho) \in \mathrm{B}\left(U^{\prime}, U^{\prime}\right)$ if and only if $\rho \geq \rho_{i}$ and $\left\|c-c_{i}\right\|^{2}=\left(\rho-\rho_{i}\right)^{2}$ for all $i$. Defining $C_{i}=c_{i}-c_{1}$, for $i=2, \ldots, m$, the latter conditon is equivalent to $\rho \geq \max _{i} \rho_{i}$ and

$$
\begin{aligned}
C^{T} C & =\left(\rho-\rho_{1}\right)^{2}, \\
\left(C_{i}-C\right)^{T}\left(C_{i}-C\right) & =\left(\rho-\rho_{i}\right)^{2} \quad(1<i \leq m) .
\end{aligned}
$$

Subtracting the latter from the former yields the $m-1$ linear equations $2 C_{i}^{T} C-C_{i}^{T} C_{i}=2 \rho\left(\rho_{i}-\rho_{1}\right)+\rho_{1}^{2}-\rho_{i}^{2}$ for $1<$ $i \leq m$. Since $c \in \operatorname{conv}\left(\left\{c_{1}, \ldots, c_{m}\right\}\right)$ by Lemma 2.2, we have $c=\sum_{i} x_{i} c_{i}$ with the $x_{i}$ 's summing up to 1 . Furthermore, 
$C=\sum x_{i}\left(c_{i}-c_{0}\right)=Q x$, where $Q=\left[C_{2}, \ldots, C_{n}\right]$ and $x=$ $\left[x_{2}, \ldots, x_{n}\right]^{T}$. Substituting this into our linear equations gives, for $1<i \leq m$,

$$
2 C_{i}^{T} Q x=C_{i}^{T} C_{i}+\rho_{1}^{2}-\rho_{i}^{2}+2 \rho\left(\rho_{i}-\rho_{1}\right) .
$$

This is a linear system of the form $A x=e+f \rho$, for $A=$ $2 Q^{T} Q$. So $B(c, \rho) \in \mathrm{B}\left(U^{\prime}, U^{\prime}\right)$ if and only if $c=Q x$, with $(x, \rho)$ being a solution of the linear system, $\rho$ a solution of $(3)$ and $\rho \geq \max _{i} \rho_{i}$. Moreover, the columns of $Q$ are linearly independent by Lemma 2.5 , so a vector $y$ is zero if and only if $Q y=\mathbf{0}$, or, equivalently, if $0=2\|Q y\|^{2}=y^{T} A y$, which shows that $A$ is regular. So we can compute $A^{-1}$, find the solution space of the linear system (which is one-dimensional) and plug this into (3). From the possible solutions $(x, \rho)$, we select the one with smallest $\rho \geq \max _{i} \rho_{i}$.

\subsection{Welzl's algorithm doesn't generalize}

Welzl's algorithm [20] for the SEBP problem can easily be 'rewritten' for balls (Figure 3). However, the resulting procedure does not work anymore in general. The reason for this is that Welzl's Lemma [20], underlying the correctness proof in the point case, fails for balls. (In section 5 we will prove the correctness of the lemma when the centers of the input balls are affinely independent, see Theorem 5.6.) A counterexample is depicted in Figure 4 (refer to Figure 2 for another): $B_{5}$ is not contained in the ball $D=\operatorname{MB}\left(\left\{B_{1}, B_{3}, B_{4}\right\},\left\{B_{1}, B_{3}, B_{4}\right\}\right)$, so the lemma suggests that $B_{5}$ be internally tangent to

$$
B^{\prime}=\operatorname{MB}\left(\left\{B_{1}, B_{3}, B_{4}, B_{5}\right\},\left\{B_{1}, B_{3}, B_{4}\right\}\right),
$$

which is not true. As a matter of fact, feeding the procedure with the balls from Figure 4 produces incorrect results from time to time, depending on the chosen permutation of the input balls. One permutation for which the algorithm fails is presented in Figure 5, where we write $\mathrm{MB}(U, V)$ as ${ }^{\prime} \mathrm{MB}(U \backslash$ $V, V)^{\prime}$ in order to save space.

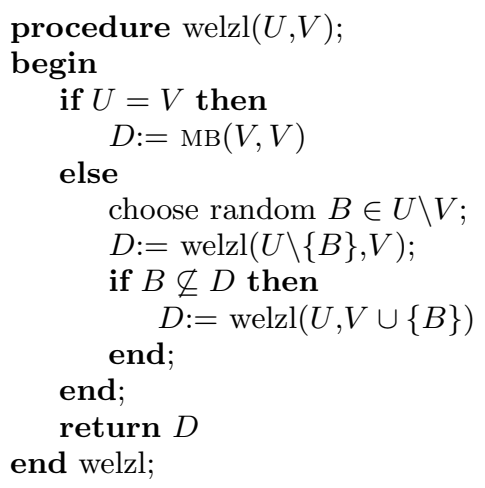

Figure 3: Welzl's algorithm for balls.

\subsection{LP-type algorithm}

From the combinatorial point of view, we want to find an inclusion-minimal set $V \subseteq U$ spanning the same miniball as $U$; since we then have $\operatorname{MB}(U)=\operatorname{MB}(V, V)$, it is straightforward to compute $\operatorname{MB}(U)$ from $V$, see Lemma 3.1. This formulation of the problem fits nicely into the framework of so-called LP-type problems [14]. Define the function

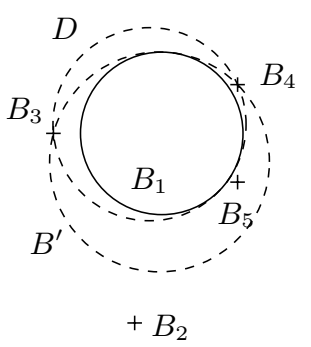

Figure 4: Balls for which Welzl's algorithm fails.

$w: 2^{U} \rightarrow \mathbb{R}^{+} \cup\{-\infty\}$, which maps a subset $U^{\prime} \subseteq U$ to the radius of $\operatorname{MB}\left(U^{\prime}\right)$, with the convention that the radius of $\operatorname{MB}(\emptyset)$ is $-\infty$. SEBB is then equivalent to finding an inclusion-minimal subset $V \subseteq U$ with $w(V)=w(U)$, and the following is easily verified using the properties from section 2 .

Lemma 3.2. For any set $U$ of balls, $(U, w)$ is an LP-type problem of combinatorial dimension $d+1$.

LP-type problems can be solved by the LP-algorithm [14], provided two primitives are available: For the violation test we are given a basis $V \subseteq U$ and a ball $B$, and we need to test whether $w(U)<w(U \cup\{B\})$. Since this is equivalent to $B \nsubseteq \mathrm{MB}(U)$, the violation test is readily implemented. The second primitive is the basis computation in which we are given a basis $V$ and a violating ball $B$ (i.e. $B \nsubseteq \mathrm{MB}(V)$ ), and we are to produce a basis of $V \cup\{B\}$. This can be solved in a brute-force manner ${ }^{3}$ by using the following primitive, the availability of which follows from Lemma 3.1.
Primitive. Given a set of at most $d+1$ balls $U$ in $\mathbb{R}^{d}$, compute the center and radius of $\operatorname{MB}(U)$ at least in the case when $U$ constitutes a basis, and signal 'no' otherwise.

In order to compute a basis of $V \cup\{B\}$, we generate all subset $V^{\prime} \subseteq V$ in increasing order of size. For each $V^{\prime}$ we invoke the primitive to test whether $U^{\prime}:=V^{\prime} \cup\{B\}$ spans $\operatorname{MB}\left(U^{\prime}\right)$, and if so, we check whether $\mathrm{MB}\left(U^{\prime}\right)$ encloses the remaining balls in $V \cup\{B\}$. The first set $V^{\prime}$ which passes the primitive and satisfies the latter containment tests constitutes, together with $B$, a basis of $V \cup\{B\}$.

Altogether, this gives an expected $2^{O(d)} n$ algorithm to compute the miniball $\mathrm{MB}(U)$ of any set of $n$ balls in $d$-space. Moreover, it is possible to do all computations in rational arithmetic (provided the input balls have rational coordinates and radii): Although the center coordinates and the radius of the miniball may have irrational coordinates, the calculations in the proof of Lemma 3.1 show that they are actually of the form $\alpha_{i}+\beta_{i} \sqrt{\gamma}$, where $\alpha_{i}, \beta_{i}, \gamma \in \mathbb{R}$ and where $\gamma \geq 0$ is the discriminant of the quadratic equation (3). Therefore, we can represent the coordinates and the radius by pairs $\left(\alpha_{i}, \beta_{i}\right) \in \mathbb{R}^{2}$, together with the discriminant $\gamma$. Since the only needed predicate is the containment test, which boils down to determining the sign of an algebraic number of degree 2, all computations can be done in $\mathbb{Q}$.

\footnotetext{
${ }^{3}$ We will improve on this in section 5. Also, Welzl's algorithm could be used here, by lifiting and subsequently perturbing the centers, but its running time is exponential, too, in some cases.
} 


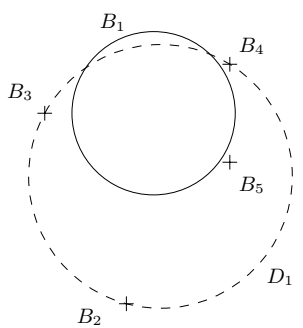

(i) In the initial call welzl $\left(\left\{B_{1}, \ldots, B_{5}\right\},\{\}\right)$, the algorithm chooses the circle $B_{1}$ and recursively computes the dashed ball $D_{1}=\operatorname{MB}\left(\left\{B_{2}, B_{3}, B_{4}, B_{5}\right\},\{\}\right)$. Then it calls welzl $\left(\left\{B_{2}, \ldots, B_{5}\right\},\left\{B_{1}\right\}\right)$ since $B_{1}$ is not contained in $D_{1}$.

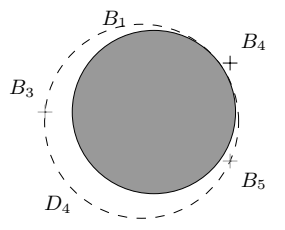

$$
B_{2}+
$$

(iv) The call welzl $\left(\{\},\left\{B_{1}, B_{3}, B_{5}\right\}\right)$ computes the dashed circle $D_{4}=\operatorname{MB}\left(\{\},\left\{B_{1}, B_{3}, B_{5}\right\}\right)$ and returns it. Back in welzl $\left(\left\{B_{4}, B_{5}\right\},\left\{B_{1}, B_{3}\right\}\right)$, the algorithm realizes that $B_{4} \notin D_{4}$ and so is forced to call welzI $\left(\left\{B_{5}\right\},\left\{B_{1}, B_{3}, B_{4}\right\}\right)$.

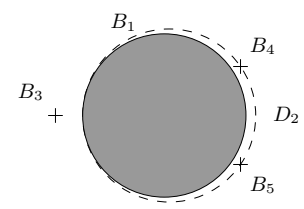

$B_{2}+$

(ii) In welzl $\left(\left\{B_{2}, \ldots, B_{5}\right\},\left\{B_{1}\right\}\right)$, the point $B_{2}$ is chosen and welzl $\left(\left\{B_{3}, B_{4}, B_{5}\right\},\left\{B_{1}\right\}\right)$ invoked. Therein, the algorithm picks $B_{3}$ and computes $D_{2}=\mathrm{MB}\left(\left\{B_{4}, B_{5}\right\},\left\{B_{1}\right\}\right)$. But $B_{3} \notin D_{2}$, so welzl $\left(\left\{B_{4}, B_{5}\right\},\left\{B_{1}, B_{3}\right\}\right)$ is called.

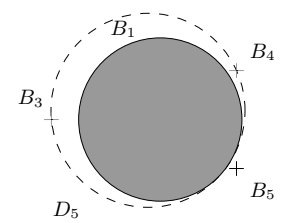

$B_{2}+$

(v) In the call welzl $\left(\left\{B_{5}\right\},\left\{B_{1}, B_{3}, B_{4}\right\}\right)$, the point $B_{5}$ is chosen and the dashed circle $D_{5}=\operatorname{MB}\left(\{\},\left\{B_{1}, B_{3}, B_{4}\right\}\right)$ is computed recursively. But again, $B_{5}$ is not contained in $D_{5}$, so welzl $\left(\{\},\left\{B_{1}, B_{3}, B_{4}, B_{5}\right\}\right)$ is invoked.

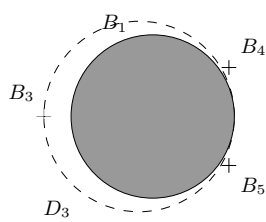

$B_{2}+$

(iii) In the call to welzl $\left(\left\{B_{4}, B_{5}\right\},\left\{B_{1}, B_{3}\right\}\right), B_{4}$ is chosen and welzl $\left(\left\{B_{5}\right\},\left\{B_{1}, B_{3}\right\}\right)$ called. Here, $B_{5}$ is evicted and the recursion yields $D_{3}=\mathrm{MB}\left(\{\},\left\{B_{1}, B_{3}\right\}\right)$. But since $B_{5} \notin D_{3}$, the algorithm calls welzl $\left(\{\},\left\{B_{1}, B_{3}, B_{5}\right\}\right)$.

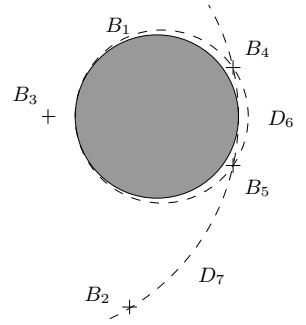

(vi) So finally welzl $\left(\{\},\left\{B_{1}, B_{3}, B_{4}, B_{5}\right\}\right)$ is called-but there doesn't exist any circle which is tangent to all these balls. The only two circles $D_{6}, D_{7}$ which are tangent to $B_{1}, B_{4}, B_{5}$ obviously don't touch $B_{3}$..

Figure 5: Five balls $\left\{B_{1}, \ldots, B_{5}\right\} \subseteq \mathbb{R}^{2}$ for which Welzl's algorithm fails.
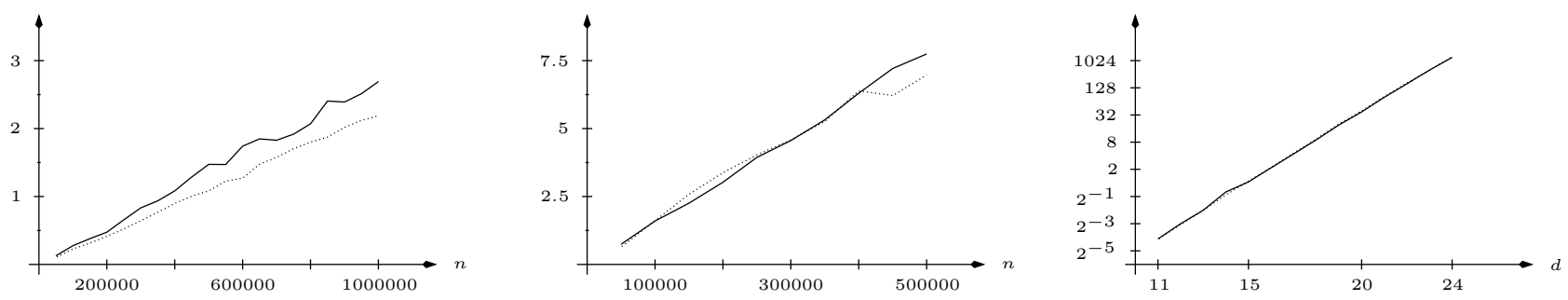

Figure 6: Running times (in seconds, averaged over 20 runs) of our heuristic in $\mathbb{R}^{3}$ (left) and $\mathbb{R}^{10}$ (middle) for $n$ balls with uniformly random center and radius (solid) and for $n$ balls chosen uniformly on the sphere with radius uniform in $[0,1 / 2]$ (dotted). The plot to the right shows the running time (in seconds, averaged over 10 runs) of the LP-algorithm (dotted) and our heuristic (solid) when fed with the vertices of the $d$-dimensional simplex. All times were measured on a $480 \mathrm{Mhz}$ Sun Ultra 4 workstation. 
We have implemented the algorithm in $\mathrm{C}++$. The code follows the generic programming paradigm and will be released with CGAL 2.5. While our implementation can be run using floating-point arithmetic, numerical problems may arise when balls are 'almost' tangent to the current miniball. In order to overcome these issues, we also provide a (deterministic) variant of the above LP-algorithm. In this heuristic - it comes without any guarantee on the running time - we maintain a basis $V$ (initially the empty set) and repeatedly add to it, by an invokation of the basis computation, the ball farthest away from the basis, that is, the ball $B$ maximizing $\delta_{V}=\max _{B \in U}\left(\left\|c-c_{B}\right\|+\rho_{B}\right), c$ being the center of $\operatorname{mB}(V)$. The algorithm stops as soon as $\delta_{V}$ is smaller or equal to the radius of $\mathrm{MB}(V)$, i.e. when all balls are contained in $\operatorname{MB}(V)$. This method, together with a suitably adapted updating-scheme from [8] to efficiently and robustly solve the equations in the proof of Lemma 3.1, handles degeneracies in a satisfying manner; numerical problems tend to only occur towards the end of the computation, when $\operatorname{MB}(V)$ is already near-optimal, and so we can simply ignore them. An extensive testsuite (similar to the one in [8]) containing various degenerate configurations of balls is passed without problems. Some running times are shown in Figures 6.

\section{INVERSION}

\subsection{Shrinking the balls}

Let $U \supseteq V$ be two sets of positive balls. Fix any ball $D \in V$ and define $s_{D}(B(c, \rho)):=B\left(c, \rho-\rho_{D}\right)$ to be the map which 'shrinks' a ball's radius by $\rho_{D}$, while keeping its center unchanged. We extend $s_{D}$ to sets $T \subseteq U$ of balls by means of $s_{D}(T)=\left\{s_{D}(B) \mid B \in T\right\}$. Recall that $\mathrm{B}(U, V)$ is the set of balls containing $U$ and being internally tangent to $V$-the following lemma implies that we can essentially restrict ourselves to the case when one of the balls in $V$ is a point.

Lemma 4.1. Let $U \supseteq V \ni D$ be two sets of positive balls. Then $B \in \mathrm{B}(U, V)$ if and only if

(i) $s_{D}(B)$ is internally tangent to the positive and externally tangent to the negative balls in $s_{D}(V)$, and

(ii) $s_{D}(B)$ contains the positive and intersects the negative balls in $s_{D}(U)$.

Proof. Let $B=B(c, \rho)$ be any ball, $\rho \geq 0$. From the definition of $\mathrm{B}(U, V)$ we have $B \in \mathrm{B}(U, V)$ if and only if

$$
\left\|c-c^{\prime}\right\| \leq \rho-\rho^{\prime}=\left(\rho-\rho_{D}\right)-\left(\rho^{\prime}-\rho_{D}\right)
$$

for all $B^{\prime} \in U$, with equality for $B^{\prime} \in V$. The claim follows immediately by observing that $\rho-\rho_{D}$ is the radius of $s_{D}(B)$ and $\rho^{\prime}-\rho_{D}$ is the radius of $s_{D}\left(B^{\prime}\right)$.

\subsection{Inversion}

We use inversion to transform a ball $B \in \mathrm{B}(U, V)$ to some simpler object. The inversion map $x^{*}:=x /\|x\|^{2}$ on $\mathbb{R}^{d} \backslash\{\mathbf{0}\}$, which we extend to point sets by $P^{*}=\left\{p^{*} \mid p \in P\right\}$ and to sets of balls by means of $U^{*}=\left\{B^{*} \mid B \in U\right\}$, has the property that containment and tangency are preserved under it. Also, a halfspace

$$
H^{+}=\left\{x \mid v^{T} x+\alpha \geq 0\right\}
$$

not containing the origin (i.e. $\alpha<0)$ maps to the ball $\left(H^{+}\right)^{*}$ with center $-v /(2 \alpha)$ and squared radius $v^{T} v /(2 \alpha)^{2}$, which has the origin on its boundary. In particular, if $v$ is a unit vector, the radius of $\left(H^{+}\right)^{*}$ is $1 /(2 \alpha)$. Conversely, a proper ball with $\mathbf{0} \in \partial B$ transforms to a halfspace not containing the origin. Furthermore, a ball $B(c, \rho)$ with $\mathbf{0} \notin B$ maps to the ball $B^{*}=B(d, \sigma)$ with

$$
d=\frac{c}{c^{2}-\rho^{2}} \quad \text { and } \quad \sigma=\frac{\rho}{c^{2}-\rho^{2}}
$$

which again doesn't contain the origin. (Refer for instance to $[2,10.8]$ and [11] for more information.)

In the sequel we denote by $H$ the hyperplane defining the halfspace $H^{+}$, and set $H^{-}:=\mathbb{R}^{d} \backslash \operatorname{ri}\left(H^{-}\right)$. Also, $H^{+}$is said to be internally (externally) tangent to a ball $B$ if and only if $B \subseteq H^{+}$(or $B \subseteq H^{-}$, respectively) and $B \cap H \neq \emptyset$.

LEMMA 4.2. Let $U \supseteq V \ni D,|U|>1$, be sets of positive balls with $c_{D}=\mathbf{0}$, no ball among them contained in another. Then $B \in \mathrm{B}(U, V)$ if and only if the halfspace $s_{D}(B)^{*}$

(i) is internally tangent to the positive and externally tangent to the negative balls in $s_{D}(V \backslash\{D\})^{*}$,

(ii) contains the positive and intersects the negative balls in $s_{D}(U \backslash\{D\})^{*}$, and

(iii) does not contain the origin.

Proof. According to Lemma 4.1, $B \in \mathrm{B}(U, V)$ if and only if $B^{\prime}:=s_{D}(B)$ is internally tangent to the positive and externally tangent to the negative balls in $V^{\prime}:=s_{D}(V)$, and if $B^{\prime}$ contains the positive and intersects the negative balls in $U^{\prime}:=s_{D}(U)$. Apply inversion w.r.t. the origin $c_{D}$. Since no ball $B_{i} \in U$ contains $D$ by assumption, $B_{i}^{\prime}:=s_{D}\left(B_{i}\right)$ does not contain the origin. (To see this, apply Lemma 4.1 to $B_{i} \notin \mathrm{B}(\{D\}, \emptyset)$.) Consequently, $B_{i}^{\prime}$ gets transformed to a ball $B\left(d_{i}, \sigma_{i}\right)$ with $d_{i}, \sigma_{i}$ as in (5). On the other hand, $B^{\prime}$ is a ball through the origin. Since $|U|>1$, its radius is strictly positive (no ball is contained in another), and hence it transforms to a halfspace $H^{+}$not containing the origin. The lemma then follows using the fact that containment and tangency are preserved under inversion.

Consider a proper (i.e. $\rho>0$ ) ball $B=B(c, \rho)$ with $\mathbf{0} \in \partial B$ and its inverse $B^{*}$, which is a halfspace of the form (4), with $v$ of unit length, say. Then the number $\alpha<0$, which represents the (signed) distance from the halfspace to the origin, is connected via $|\rho|=-1 /(2 \alpha)$ to the radius of $B$ : that is, radius and distance are reciprocal to each other (if we neglect the factor -2$)$. It follows that $B$ is the smallest ball in $\mathrm{B}(U, V)$, i.e. $B=\operatorname{MB}(U, V)$, if and only if the hyperplane $s_{D}(B)^{*}$ is, among the hyperplanes satisfying (i-iii), the one with maximal (absolute) distance $-\alpha$ to the origin. An example is shown in Figure 7. For some set $U=\left\{B_{1}, \ldots, B_{5}\right\}$ of five balls, the shrunken balls $S_{i}:=s_{B_{1}}\left(B_{i}\right)$ are depicted, $B_{i} \in U$; positive balls with solid, negative balls with dotted border. The right-hand side of the figure shows the configuration after inversion w.r.t. the center $c_{1}$ of $B_{1}$. As a dashed ball you can see $B:=s_{B_{1}}\left(\operatorname{MB}\left(U,\left\{B_{3}\right\}\right)\right.$ ) (left) which corresponds to the dashed halfspace in the right picture. This halfspace is the 'farthest' among the halfspaces which contain $S_{5}^{*}$, intersect $S_{2}^{*}$ and $S_{4}^{*}$, are internally tangent to $S_{3}^{*}$ and do not contain the origin.

Observe that a halfspace $\mathrm{H}^{+}$of the form (4) is internally (externally) tangent to the positive (negative) ball $B(d, \sigma)$ if and only if $\left(v^{T} d+\alpha\right) /\|v\|=\sigma$, and that $H^{+}$contains (intersects) the positive (negative) ball $B(d, \sigma)$ if and only if $\left(v^{T} d+\alpha\right) /\|v\| \geq \sigma$. This together with Lemma 4.2 gives: 

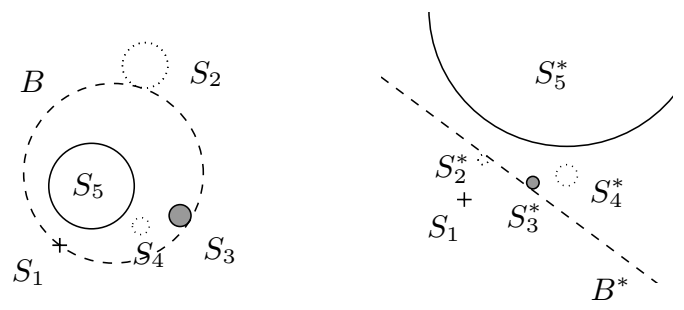

Figure 7: Example to Lemma 4.2.

LemmA 4.3. Let $U \supseteq V \ni D,|U|>1$, be sets of positive balls with $c_{D}=\mathbf{0}$, no ball in another. Then $B^{\prime} \in \mathrm{B}(U, V)$ if and only if

$$
B^{\prime}=B(-v /(2 \alpha),-1 /(2 \alpha)),
$$

where $(v, \alpha) \in \mathbb{R}^{d+1}$ with $\alpha<0$ is a feasible point of the following program.

$$
\begin{aligned}
& P_{D}(U, V) \text { minimize } \alpha \\
& \text { subject to } d_{B}^{T} v+\alpha \geq \sigma_{B}, \quad B \in U \backslash V \text {, } \\
& d_{B}^{T} v+\alpha=\sigma_{B}, \quad D \neq B \in V,
\end{aligned}
$$

Moreover, $B^{\prime} \in \operatorname{MB}(U, V)$ if and only if $(v, \alpha)$ is an optimal solution to $P_{D}(U, V)$.

\subsection{The distance to the convex hull}

As shown in [7], the SEBP problem can be reduced to the problem DHP of finding the distance from a given point to the convex hull of a point set $P$ (together with the point in $\operatorname{conv}(P)$ where this distance is attained). The reduction is based on the following fact, which holds for points but is not true in general for balls (see Figure 8, where the point in $\operatorname{conv}\left(\left\{c_{1}, c_{2}, c_{3}\right\}\right)$ (gray) closest to the circumcenter $c$ does not coincide with the center of the miniball; the dotted line constitutes the centers of all balls tangent to $B_{1}$ and $B_{2}$ ).

Lemma 4.4. Let $P \subseteq \mathbb{R}^{d}$ be a set of affinely independent points with circumcenter $c^{\prime}$. The center of the ball $\mathrm{MB}(P)$ is the point in $\operatorname{conv}(P)$ with minimal distance to $c^{\prime}$.

The miniball of $d+2$ points can thus be found by computing the smallest balls spanned by any subset of $d+1$ points, that is, by solving $d+2$ instances of DHP. Using the material from the previous section, we can give a similar reduction from SEBB to DHB, the problem of finding the distance from a given point to the convex hull of a set of balls. For this, define $\operatorname{MB}_{p}(U):=\operatorname{MB}(U \cup\{p\},\{p\})$ to be the smallest ball enclosing the balls $U \not \supset p$ while having a given point $p \in \mathbb{R}^{d}$ on its boundary.

LEMMA 4.5. DHB is equivalent to the problem of finding $\mathrm{MB}_{p}(U)$ for given (positive) balls $U$ and a point $p \in \mathbb{R}^{d}$.

Proof. We transform an instance $(U, p)$ of DHB to an instance $\left(U^{\prime}, p^{\prime}\right)$ of $\mathrm{MB}_{p^{\prime}}\left(U^{\prime}\right)$ and show how the solution to the former relates to the solution of the latter. In doing so, we assume that the input ball sets $U$ (or $U^{\prime}$, respectively) are such that no ball is contained in another one; enclosed balls can be removed in a preprocessing step without affecting the solution. Furthermore, we can assume $p=\mathbf{0}$ (or $p^{\prime}=\mathbf{0}$, respectively) which is always achieved by a suitable translation. Consider inversion w.r.t. the ball $D:=\{p\}$ of radius 0 and consider $U^{\prime}:=U^{*}$, which then is a set of balls, none among them contained in another. Since $s_{D}(B)=B$ for any ball $B$, Lemma 4.2 states that $B \in \mathrm{B}(U \cup\{D\},\{D\})$ if and only if the halfspace $H^{+}:=B^{*}$ contains all balls $U^{\prime}$ but not the origin. Or, equivalently, $B \in \mathrm{B}(U \cup\{D\},\{D\})$ if and only if $H$ is a hyperplane strictly separating the origin from $\operatorname{conv}\left(U^{\prime}\right)$. Write

$$
H^{+}=\left\{x \mid v^{T} x+\alpha \geq 0\right\}
$$

with $\|v\|=1$, and recall that the radius of $B$ is $\rho=-1 /(2 \alpha)$. This implies that $\operatorname{MB}_{p}(U)^{*}$ is the farthest among the hyperplanes which strictly separate $\operatorname{conv}\left(U^{\prime}\right)$ from the origin, i.e. the one with maximal distance to the origin. It is easily verified that $H$ with $\alpha<0$ is the farthest among the strictly separating hyperplanes if and only if $-\alpha$ equals the distance from conv $\left(U^{\prime}\right)$ to the origin. (The distance is zero if and only if there doesn't exist any strictly separating hyperplane.) Consequently, $\operatorname{MB}_{p}(U)$ is infeasible if and only if $\min _{p \in \operatorname{conv}\left(U^{\prime}\right)}\|p\|=0$, and a ball $B$ coincides with $\operatorname{MB}_{p}(U)$ if and only if the halfspace $B^{*}$ has distance $\min _{p \in \operatorname{conv}\left(U^{\prime}\right)}\|p\|$ to the origin.

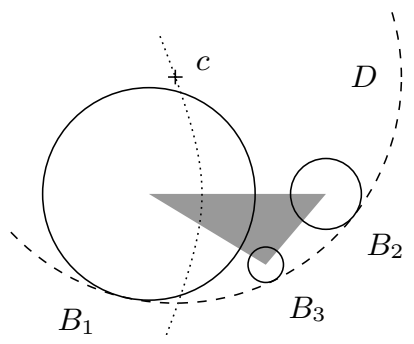

Figure 8: Lemma 4.4 doesn't hold for balls.

The equivalence of DHB and SEBB now follows from the fact that SEBB and the problem of finding $\operatorname{MB}_{p}(U)$ for given point $p \in \mathbb{R}^{d}$ are equivalent; the latter will be shown in the next section, where we also develop an algorithm to find $\operatorname{MB}_{p}(U)$. We remark that $\operatorname{MB}_{p}(U)$ can also be computed by a suitably modified version of the algorithm from section 3.2.

\section{SMALL CASES REVISITED}

We have introduced two methods for computing $\operatorname{MB}(U)$ if $U$ is small (meaning $|U| \leq d+2$ ). The method we have implemented systematically searches for a basis $V \subseteq U$, a minimal subset such that $\operatorname{MB}(U)=\mathrm{MB}(V, V)$. Another approach, the correctness of which will be discussed below, embeds $U$ into $\mathbb{R}^{d+1}$, (symbolically) perturbs the balls in $U$ to make their centers affinely independent and then runs Welzl's algorithm which also computes a basis $V$. However, it is not hard to construct inputs for which both approaches need to examine $\Omega\left(2^{d}\right)$ sets $V$ in order to find the basis of $U$. A goal of this section is to demonstrate that one can improve on this, by showing that the problem has more structure than we have exploited so far.

In our approach we reduce the problem of finding $\operatorname{MB}(U)$ to that of computing $\operatorname{MB}_{p}(W)$ for some other set $W$ of balls: To obtain $\operatorname{MB}(U)$, we 'guess' the smallest ball $B \in U$ tangent to $\operatorname{MB}(U)$, find the set $W$ of positive shrunken balls,

$$
W=\left\{B^{\prime} \in s_{B}(U \backslash\{B\}) \mid B^{\prime} \text { positive }\right\},
$$


and compute $\mathrm{MB}_{c_{B}}(W)=s_{B}(\mathrm{MB}(U))$ from which $\operatorname{MB}(U)$ is easily reconstructed (Lemma 4.1). This takes at most $|U|$ guesses and therefore introduces only polynomial overhead.

Our method to find $\mathrm{MB}_{p}(T), T$ a set of positive balls, computes as intermediate steps balls of the form $\operatorname{MB}_{p}\left(U^{\prime}, V^{\prime}\right):=$ $\operatorname{MB}\left(U^{\prime} \cup\{p\}, V^{\prime} \cup\{p\}\right)$, for $V^{\prime} \subseteq U^{\prime} \subseteq T$. One obstacle we have to overcome for this is the possible nonexistence of $\operatorname{MB}_{p}\left(U^{\prime}, V^{\prime}\right)$ even in the case where the centers of the balls in $T$ are affinely independent (we have seen an example). Our solution employs the inversion transformation: With respect to the given point $p$, it defines for all triples $\left(p, U^{\prime}, V^{\prime}\right)$ a 'generalized ball' $\operatorname{GMB}_{p}\left(U^{\prime}, V^{\prime}\right)$ which is always defined and coincides with $\operatorname{MB}_{p}\left(U^{\prime}, V^{\prime}\right)$ if the latter exists.

Subsequently we prove that a unique sink orientation [19] can be imposed on the $|T|$-dimensional cube graph whose vertices are associated with the sets $2^{T}$. The crucial property of this orientation is that $\operatorname{GMB}_{p}\left(T^{\prime}, T^{\prime}\right)=\operatorname{GMB}_{p}(T, \emptyset)$ holds for its sink $T^{\prime}$. Moreover, the edge orientations are easily computable, with the consequence that the algorithms in [19] can be used to compute $\operatorname{GMB}_{p}(T, \emptyset)$ (and hence $\operatorname{MB}(U)$ ) in expected time $O\left(c^{d}\right)$ for some constant $c<2$ (currently, $c=1.4 \ldots$ is the best upper bound). Another attractive feature of the unique sink aproach is that it allows pivoting methods which may not be worst-case efficient butunlike the two methods discussed above- have the potential of finding the solution very fast in practice.

For the rest of this section, we fix a point $p \in \mathbb{R}^{d}$ and a set $T$ of balls, and assume that the centers of $T \cup\{p\}$ are affinely independent and that none among them is contained in another (it follows that $|T| \leq d$ ). By translation of the input, we can furthermore assume $p=\mathbf{0}$ w.l.o.g. Performing inversion w.r.t. $p$ as described in the previous section gives us $|T|$ balls $T^{*}$ with linearly independent centers $d_{B}$ and radii $\sigma_{B}, B \in T$. The following lemma is then an easy consequence of previous considerations.

Lemma 5.1. For given $V \subseteq U \subseteq T$ with $|U| \geq 1$, consider the following (nonconvex) optimization problem in the variables $v \in \mathbb{R}^{d}, \alpha \in \mathbb{R}$.

$$
\begin{aligned}
& \mathcal{P}_{p}(U, V) \text { lexmin } \quad\left(v^{T} v, \alpha\right), \\
& \text { subject to } d_{B}^{T} v+\alpha \geq \sigma_{B}, \quad B \in U \backslash V \text {, } \\
& d_{B}^{T} v+\alpha=\sigma_{B}, \quad B \in V, \\
& v^{T} v \geq 1
\end{aligned}
$$

(i) $\mathcal{P}_{p}(U, V)$ has a unique optimal solution $(\tilde{v}, \tilde{\alpha})$.

(ii) If $\mathrm{MB}_{p}(U, V)$ exists, then $(\tilde{v}, \tilde{\alpha})$ is an optimal solution to $P_{D}(U \cup\{D\}, V \cup\{D\})$ from section 4.2 for $D:=\{p\}$.

Proof. (i) Because the $d_{B}, B \in U \subseteq T$, are linearly independent, there is a vector $w$ (which we call an unbounded direction) such that

$$
d_{B}^{T} w=1, \quad B \in U
$$

Now assume that $\mathcal{P}_{p}(U, V)$ has two distinct optimal solutions $\left(\tilde{v}_{1}, \tilde{\alpha}\right),\left(\tilde{v}_{2}, \tilde{\alpha}\right), \tilde{v}_{1}^{T} \tilde{v}_{1}=\tilde{v}_{2}^{T} \tilde{v}_{2}=\delta \geq 1$. Consider any proper convex combination $v$ of $\tilde{v}_{1}$ and $\tilde{v_{2}} ; v$ satisfies $v^{T} v<$ $\delta$. Then there is a suitable positive constant $\Theta$ such that $(v+\Theta w)^{T}(v+\Theta w)=\delta$, and hence the pair $(v+\Theta w, \tilde{\alpha}-\Theta)$ is a feasible solution to $\mathcal{P}_{p}(U, V)$, a contradiction to lexicographic minimality of the initial solutions. (ii) If $\operatorname{MB}_{p}(U, V)$ exists, the program $P_{D}(U \cup\{D\}, V \cup\{D\})$, has a feasible solution and is therefore equivalent to $\mathcal{P}_{p}(U, V)$.
Definition 5.2. For $V \subseteq U \subseteq T$, let $\operatorname{GMB}_{p}(U, V)$ denote the ball of radius $\rho$ and center $c$,

$$
\rho=-1 /(2 \tilde{\alpha}) \quad \text { and } \quad c=\tilde{v} \rho,
$$

where the pair $(\tilde{v}, \tilde{\alpha})$, which we call the value of $\operatorname{GMB}_{p}(U, V)$, is the optimal solution to $\mathcal{P}_{p}(U, V)$; for $U=\emptyset$, we define $\operatorname{GMB}_{p}(U, V):=\{p\}$ with value $(\mathbf{0},-\infty)$.

Notice that by Lemma 5.1 and $4.3, \operatorname{GMB}_{p}(U, V)$ coincides with $\operatorname{MB}_{p}(U, V)$ in case the latter exists.-Although $\mathcal{P}_{p}(U, V)$ is not a convex program, it is equivalent to one of the two related convex programs given below: $\mathcal{C}_{p}^{\prime}(U, V)$ finds the lowest point in a cylinder, subject to linear (in)equality constraints; in case it doesn't have solution (i.e. if $\operatorname{MB}_{p}(U, V)$ doesn't exist), the other program $\mathcal{C}_{p}(U, V)$ applies in which the cylinder is allowed to enlarge until the feasibilty region becomes non-empty.

LEMma 5.3. Let $(\tilde{v}, \tilde{\alpha})$ be the optimal solution to program $\mathcal{P}_{p}(U, V)$, for $|U| \geq 1$, and let $\gamma$ be the minimum value of the quadratic program

$$
\begin{array}{lll}
\mathcal{C}_{p}(U, V) \quad \begin{array}{l}
\text { minimize } \\
\text { subject to } \quad
\end{array} & d_{B}^{T} v+\alpha \geq \sigma_{B}, \quad B \in U \backslash V, \\
& d_{B}^{T} v+\alpha=\sigma_{B}, \quad B \in V .
\end{array}
$$

(i) If $\gamma \geq 1$, then $\mathcal{P}_{p}(U, V)$ and $\mathcal{C}_{p}(U, V)$ have the same, unique solution $(\tilde{v}, \tilde{\alpha})$.

(ii) If $\gamma<1$, then $\tilde{v}^{T} \tilde{v}=1$ and $(\tilde{v}, \tilde{\alpha})$ is the unique optimal solution to the convex program

$$
\begin{aligned}
\mathcal{C}_{p}^{\prime}(U, V) \text { minimize } & \alpha \\
\text { subject to } & d_{B}^{T} v+\alpha \geq \sigma_{B}, \quad B \in U \backslash V, \\
& d_{B}^{T} v+\alpha=\sigma_{B}, \quad B \in V, \\
& v^{T} v \leq 1 .
\end{aligned}
$$

Also, $\mathcal{C}_{p}(U, V)$ is strictly feasible (i.e. feasible values $(v, \alpha)$ exist such that all inequality constraints are satisfied with strict inequality). If $\gamma<1, \mathcal{C}_{p}^{\prime}(U, V)$ is strictly feasible, too.

Proof. (i) Using an unbounded direction (6) as in the proof of the previous lemma, it is easy to see that $\mathcal{C}_{p}(U, V)$ has a unique optimal vector $v$ which in turn uniquely determines $\alpha$, because $\gamma \geq 1$ implies $V \neq \emptyset$. Given this, the programs $\mathcal{P}_{p}(U, V)$ and $\overline{\mathcal{C}}_{p}(U, V)$ are obviously equivalent under $\gamma \geq 1$. (ii) Again using the unbounded direction (6), we can show that the optimal vector $v$ in $\mathcal{C}_{p}^{\prime}(U, V)$ is unique and satisfies $v^{T} v=1$; the equivalence of $\mathcal{P}_{p}(U, V)$ and $\mathcal{C}_{p}^{\prime}(U, V)$ under $\gamma<1$ follows.

To see strict feasibility of $\mathcal{C}_{p}^{\prime}(U, V)$, first note that $\gamma<$ 1 implies the existence of a feasible pair $(v, \alpha)$ for which $v^{T} v<1$. Linear independence of the $d_{B}$ yields a vector $w^{\prime}$ such that

$$
d_{B}^{T} w^{\prime}=\left\{\begin{array}{ll}
1, & B \in U \backslash V, \\
0, & B \in V
\end{array} .\right.
$$

For a suitable small $\Theta>0$, the pair $\left(v+\Theta w^{\prime}, \alpha\right)$ is strictly feasible for $\mathcal{C}_{p}^{\prime}(U, V)$. Strict feasibility of $\mathcal{C}_{p}(U, V)$ follows by an even simpler proof along these lines.

The following characterization of optimality is an application of the Karush-Kuhn-Tucker conditions for convex programming [1, Theorem 5.3.1 with Slater's constraint qualification together with Theorem 4.3.8]. 
Theorem 5.4. Let $V \subseteq U \subseteq T$ with $|U| \geq 1$.

(i) A feasible solution $(\tilde{v}, \tilde{\alpha})$ to $\mathcal{C}_{p}(U, V)$ is optimal if and only if there exist (unique) real numbers $\lambda_{B}, B \in U$, such that

$$
\begin{aligned}
\lambda_{B} & \geq 0, \quad B \in U \backslash V \\
\lambda_{B}\left(d_{B}^{T} \tilde{v}+\tilde{\alpha}-\sigma_{B}\right) & =0, \quad B \in U \backslash V, \\
\sum_{B \in U} \lambda_{B} d_{B} & =\tilde{v}, \\
\sum_{B \in U} \lambda_{B} & =0 .
\end{aligned}
$$

(ii) A feasible solution $(\tilde{v}, \tilde{\alpha})$ to $\mathcal{C}_{p}^{\prime}(U, V)$ satisfying $\tilde{v}^{T} \tilde{v}=$ 1 is optimal if and only if there exist (unique) real numbers $\lambda_{B}, B \in U$, such that

$$
\begin{aligned}
\lambda_{B} & \geq 0, \quad B \in U \backslash V \\
\lambda_{B}\left(d_{B}^{T} \tilde{v}+\tilde{\alpha}-\sigma_{B}\right) & =0, \quad B \in U \backslash V, \\
\sum_{B \in U} \lambda_{B} d_{B} & =\tilde{v} \\
\sum_{B \in U} \lambda_{B} & >0 .
\end{aligned}
$$

We remark that under affine independence Lemma 2.2 is a special case of this theorem. Also, we can use these optimality conditions to state a version of Welzl's Lemma 1 [20] for $\operatorname{GMB}_{p}(U, V)$.

Lemma 5.5. Let $V \subseteq U \subseteq T$ and $B \in U \backslash V$. Denote by $(\tilde{v}, \tilde{\alpha})$ the value of $\operatorname{GMB}_{p}(U \backslash\{B\}, V)$. Then

$$
\operatorname{GMB}_{p}(U, V)= \begin{cases}\operatorname{GMB}_{p}(U \backslash\{B\}, V), & \text { if } d_{B}^{T} \tilde{v}+\tilde{\alpha} \geq \sigma_{B}, \\ \operatorname{GMB}_{p}(U, V \cup\{B\}), & \text { otherwise. }\end{cases}
$$

Proof. The case $U=\{B\}$ is easily checked directly, so assume $|U|>1$. If $d_{B}^{T} \tilde{v}+\tilde{\alpha} \geq \sigma_{B}$, then $(\tilde{v}, \tilde{\alpha})$ is feasible and hence optimal for the more restricted problem $\mathcal{P}_{p}(U, V)$. Otherwise, the value $\left(\tilde{v}^{\prime}, \tilde{\alpha}^{\prime}\right)$ of $\operatorname{GMB}_{p}(U, V)$ is definitely different from $(\tilde{v}, \tilde{\alpha})$. Now consider the coefficient $\lambda_{B}$ resulting from the application of Theorem 5.4 to $\left(\tilde{v}^{\prime}, \tilde{\alpha}^{\prime}\right)$, which by Lemma 5.3 is the optimal solution to $\mathcal{C}_{p}(U, V)$ or $\mathcal{C}_{p}^{\prime}(U, V)$. We must have $\lambda_{B} \neq 0$, because Theorem 5.4 would otherwise certify that $\left(\tilde{v}^{\prime}, \tilde{\alpha}^{\prime}\right)$ is optimal for $(U \backslash\{B\}, V)$. This, however, implies that

$$
d_{B}^{T} \tilde{v}^{\prime}+\tilde{\alpha}^{\prime}=\sigma_{B}
$$

and $\operatorname{GMB}_{p}(U, V)=\operatorname{GMB}_{p}(U, V \cup\{B\})$ follows.

When $\operatorname{MB}_{p}(U, V)$ (and hence $\operatorname{MB}_{p}(U \backslash\{B\}, V)$ ) exists, the test $d_{B}^{T} \tilde{v}+\tilde{\alpha} \geq \sigma_{B}$ is nothing but a containment test $B \subseteq$ $\operatorname{MB}_{p}(U \backslash\{B\}, V)$. Using a shrinking argument as in section 4.1, we can thus show Welzl's Lemma for balls, and it follows that Welzl's algorithm (Section 3) computes $\operatorname{MB}(U)$ for a set of balls whose centers are affinely independent:

THEOREM 5.6. Let $V \subseteq U \subseteq T$, where $T$ is a set of balls, none contained in another, with affinely independent centers. If $\operatorname{mB}(U, V)$ exists and $B \in U \backslash V$ with

$$
B \nsubseteq \operatorname{MB}(U \backslash\{B\}, V),
$$

then $\operatorname{MB}(U, V)=\operatorname{MB}(U, V \cup\{B\})$.

Proof. For $V=\emptyset$, the claim follows by the very same argument as in Welzl's original lemma [20]. In case of $|V|=$ 1 , we shrink all balls by the radius of the ball in $V$ and again use convex combinations as in Welzl's proof, together with Lemma 4.1.
When $|V|>1$, we fix $B \in V$ and shrink all involved balls by $\rho_{B}$, yielding the set $s_{B}(U)$ which possibly contains negative balls. Observe that the material from this section also holds for sets $T$ with possibly negative radii, when $\operatorname{MB}_{p}(U, V)$ is defined to be the smallest ball tangent to $p$ which contains (intersects) the positive (negative) balls in $U$ and is internally (externally) tangent to the positive (negative) balls in $V$. The claim then follows from Lemma 5.5.

\subsection{The unique sink orientation}

As in the previous section, we consider a point $p \in \mathbb{R}^{d}$ ( $p=\mathbf{0}$ w.l.o.g.) and a set $T=\left\{B_{1}, \ldots, B_{n}\right\}$ of at most $d$ balls such that the centers of $T \cup\{p\}$ are affinely independent and such that no ball is contained in another. Look at the $n$-dimensional cube. Its vertices can be identified with the subsets $J \subseteq I:=\{1, \ldots, n\}$; faces of the cube then correspond to pairs $[F, G]:=\{H \mid F \subseteq H \subseteq G\}$, where $F \subseteq G \subseteq I$. We consider the cube graph

$$
G=\left(2^{I},\left\{(J, J \oplus\{i\}) \mid J \in 2^{I}, i \in I\right\}\right),
$$

where $\oplus$ denotes symmetric difference between sets. An orientation $\mathcal{O}$ of the edges of $G$ is called a unique sink orientation if for any face $[F, G]$, the subgraph of $G$ induced by the vertices of $[F, G]$ has a unique sink w.r.t. $\mathcal{O}[19]$.

We associate to a vertex $J \subseteq I$ of the cube the set of balls

$$
T_{J}:=\left\{B_{i} \mid i \in J\right\} \subseteq T,
$$

and write in the rest of this section $\operatorname{GMB}_{p}(G, F)$ as a shorthand for $\operatorname{GMB}_{p}\left(T_{G}, T_{F}\right)$, for any $F \subseteq G \subseteq I$. Moreover, we write $d_{i}:=d_{B_{i}}$ and $\sigma_{i}:=\sigma_{B_{i}}$ for the center and radius of the inverted balls $B_{i}^{*} \in T^{*}$.- The following is the main result of this section.

TheOrem 5.7. Consider the orientation $\mathcal{O}$ defined by

$$
J \rightarrow J \cup\{i\} \quad: \Leftrightarrow \operatorname{GMB}_{p}(J, J) \neq \operatorname{GMB}_{p}(J \cup\{i\}, J) .
$$

Then $\mathcal{O}$ is a unique sink orientation, and the sink $J$ of the cube is a basis of $\operatorname{GMB}_{p}(I, \emptyset)$, meaning that $J$ is inclusionminimal with $\operatorname{GMB}_{p}(J, J)=\operatorname{GMB}_{p}(I, \emptyset)$.

Proof. Fix an arbitrary face $[F, G]$ of the cube and let $\lambda_{i}, i \in G$, be the multipliers guaranteed by Theorem 5.4 for the value $(\tilde{v}, \tilde{\alpha})$ of $\operatorname{GMB}_{p}(G, F)$, with $\mathcal{D} \in\left\{\mathcal{C}_{p}, \mathcal{C}_{p}^{\prime}\right\}$ being the relevant program. Define $J$ to be the set

$$
F \cup\left\{i \in G \mid d_{i}^{T} \tilde{v}+\tilde{\alpha}=\sigma_{i}, \lambda_{i} \neq 0\right\} .
$$

We first prove that $J$ is a sink in the face $[F, G]$ and that it is inclusion-minimal with

$$
\operatorname{GMB}_{p}(G, F)=\operatorname{GMB}_{p}(J, J) .
$$

Since $\lambda_{i}=0$ for all $i \in G \backslash J,(\tilde{v}, \tilde{\alpha})$ is a solution to program $\mathcal{D}(J, J)$ by Theorem 5.4 and hence (8) holds. By the very same argument, the pair also solves $\mathcal{D}(J \cup\{i\}, J)$ for any such $i$, and hence

$$
\operatorname{GMB}_{p}(J, J)=\operatorname{GMB}_{p}(J \cup\{i\}, J), \quad i \in G \backslash J .
$$

Moreover, (8) implies $\lambda_{i} \geq 0$ for $i \in G \backslash F$ and hence $(\tilde{v}, \tilde{\alpha})$ is a solution to $\mathcal{D}(J, F)$, too. Consequently,

$$
\operatorname{GMB}_{p}(J, F)=\operatorname{GMB}_{p}(J, J),
$$

from which it follows that $\operatorname{GMB}_{p}(J, J \backslash\{i\})=\operatorname{GMB}_{p}(J, J)$ for any $i \in J \backslash F$ (to see this, observe that the programs $\mathcal{D}(J, F)$, $\mathcal{D}(J, J \backslash\{i\})$ and $\mathcal{D}(J, J)$ are more and more restrictive, with 
$(\tilde{v}, \tilde{\alpha})$ a solution to both the first and the last by the above). So $(\tilde{v}, \tilde{\alpha})$ solves $\mathcal{D}(J, J \backslash\{i\})$ and consequently

$$
\operatorname{GMB}_{p}(J \backslash\{i\}, J \backslash\{i\}) \neq \operatorname{GMB}_{p}(J, J \backslash\{i\}), i \in J \backslash F,
$$

because $\lambda_{i} \neq 0$ by definition of $J$. This together with (9) proves $J$ to be a sink of $[F, G]$ and shows that $J$ is inclusionminimal such that $(8)$ holds.

As to uniqueness, let $J \in[F, G]$ be any sink; we show that $J$ is of the form (7). Let $(\tilde{v}, \tilde{\alpha})$ be the value of $\operatorname{GMB}_{p}(J, J)$, with $\mathcal{D} \in\left\{\mathcal{C}_{p}, \mathcal{C}_{p}^{\prime}\right\}$ the relevant program. $J$ being a sink implies (9) and (10), and from the latter and Lemma 5.5 it follows that $\operatorname{GMB}_{p}(J, J)=\operatorname{GMB}_{p}(J, J \backslash\{i\})$ for any $i \in J \backslash F$. If we again denote by $\lambda_{i}, i \in J$, the multipliers of $(\tilde{v}, \tilde{\alpha})$ which are guaranteed by Theorem 5.4, we see that $\lambda_{i} \geq 0$ for all $i \in J \backslash F$; moreover, $\lambda_{i}>0$ by (10), so that Theorem 5.4 yields $d_{i}^{T} \tilde{v}+\tilde{\alpha}=\sigma_{i}$ for all $i \in J \backslash F$. It remains to show that $i \in J$ for all $i \in G$ with $\lambda_{i} \neq 0$ and $d_{i}^{T} \tilde{v}+\tilde{\alpha}=\sigma_{i}$. For this, observe that (9) implies $\lambda_{i}=0$ for all $i \in G \backslash J$.

Specialized to the case of points, this result already follows from Lemma 5 in [9]. However, our proof shows that the general position assumption made for this in [9] is not necessary, because affine independence already yields the degree of general position that we need.

In order to apply USO algorithms (e.g. the ones in [19]) to find the sink of our orientation $\mathcal{O}$, we have to evaluate the orientation of an edge $(J, J \oplus\{i\})$, i.e. we must check

$$
\operatorname{GMB}_{p}(J, J) \neq \operatorname{GMB}_{p}(J \cup\{i\}, J) .
$$

For this, we first solve $\mathcal{C}_{p}(J, J)$ (which amounts to solving a regular system of linear equations by Theorem 5.4). If the solution $(\tilde{v}, \tilde{\alpha})$ satisfies $\gamma:=\tilde{v}^{T} \tilde{v} \geq 1$, we have already found the value $(\tilde{v}, \tilde{\alpha})$ of $\operatorname{GMB}_{p}(J, J)$, and we simply evaluate

$$
d_{i}^{T} \tilde{v}+\tilde{\alpha} \geq \sigma_{i},
$$

which then is equivalent to (11). If $\gamma<1, \operatorname{GMB}_{p}(J, J)$ coincides with $D:=\operatorname{MB}_{p}(J, J)$ which we compute by means of Lemma 3.1 ; here, (11) is equivalent to $B_{i} \nsubseteq D$.

\section{CONCLUSIONS \& OPEN PROBLEMS}

There are recent, practically efficient algorithms for SEBB which in polynomial time compute a $(1+\varepsilon)$-approximation [12]. While exact algorithms with subexponential complexity exist for SEBP [7], we have so far not been able to give one for SEBB (or DHB, equivalently). Another interesting question is how existing pivoting rules work when applied to our USO formulation from Section 5; we haven't done any experiments yet but expect a significant speedup in practice.

\section{ACKNOWLEDGEMENT}

We thank Menelaos Karavelas for pointing out the usefulness of the inversion transformation in this context.

\section{REFERENCES}

[1] M. S. Bazaraa, H. D. Sherali, and C. M. Shetty. Nonlinear programming: theory and applications. Wiley, 1979.

[2] M. Berger. Geometry (vols. 1-2). Springer-Verlag, 1987.

[3] B. Chazelle and J. Matoušek. On linear-time deterministic algorithms for optimization problems in fixed dimension. J. Algorithms, 21:579-597, 1996.
[4] V. Chvátal. Linear programming. W. H. Freeman, New York, NY, 1983.

[5] M. E. Dyer. Linear time algorithms for two- and three-variable linear programs. SIAM J. Comput., 13:31-45, 1984.

[6] M. E. Dyer. A class of convex programs with applications to computational geometry. In Proc. 8th Annu. ACM Sympos. Comput. Geom., pages 9-15, 1992.

[7] B. Gärtner. A subexponential algorithm for abstract optimization problems. SIAM J. Comput, 24(5):1018-1035, 1995.

[8] B. Gärtner. Fast and robust smallest enclosing balls. In Proc. 7th Annual European Symposium on Algorithms (ESA), volume 1643 of Lecture Notes Comput. Sci., pages 325-338. Springer-Verlag, 1999.

[9] B. Gärtner and E. Welzl. Explicit and implict enforcing - randomized optimization. In Computational Discrete Mathematics, volume 2122 of LNCS, pages 25-46. Springer-Verlag, 2001.

[10] T. H. Hopp and C. P. Reeve. An algorithm for computing the minimum covering sphere in any dimension. Technical Report NISTIR 5831, National Institute of Standards and Technology, 1996.

[11] M. Karavelas and I. Emiris. Predicates for the planar additively weighted Voronoi diagram. ECG Technical Report ECG-TR-122201-01, Sophia-Antipolis, 2002.

[12] P. Kumar, J. S. B. Mitchell, and E. A. Yıldırım. Computing core-sets and approximate smallest enclosing hyperspheres in high dimensions. To appear in the Proceedings of ALENEX'03.

[13] R. Kurniawati, J. S. Jin, and J. A. Shepherd. The $\mathrm{SS}^{+}$-tree: an improved index structure for similarity searches in a high-dimensional feature space. In Proc. 5th Storage and Retrieval for Image and Video Databases SPIE, volume 3022, pages 110-120, 1997.

[14] J. Matoušek, M. Sharir, and E. Welzl. A subexponential bound for linear programming. Algorithmica, 16:498-516, 1996.

[15] N. Megiddo. Linear-time algorithms for linear programming in $R^{3}$ and related problems. SIAM J. Comput., 12(4):759-776, 1983.

[16] N. Megiddo. Linear programming in linear time when the dimension is fixed. J. ACM, 31:114-127, 1984.

[17] N. Megiddo. On the ball spanned by balls. Discrete Comput. Geom., 4:605-610, 1989.

[18] R. Seidel. e-mail communication, 1997.

[19] T. Szabó and E. Welzl. Unique sink orientations of cubes. In Proc. 42nd annual IEEE Symposium on Foundations of Computer Science (FOCS), pages 547-555, 2001.

[20] E. Welzl. Smallest enclosing disks (balls and ellipsoids). In H. Maurer, editor, New Results and New Trends in Computer Science, volume 555 of Lecture Notes Comput. Sci., pages 359-370. Springer-Verlag, 1991.

[21] H.-M. Will. Computation of additively weighted Voronoi cells for applications in molecular biology. PhD thesis, ETH Zürich, 1998. 